\title{
Structural and semantic ambiguity of $w h y$-questions: An overlooked case of weak islands in English
}

\author{
Cassandra Chapman \& Ivona Kučerová*
}

\begin{abstract}
We argue that English why-questions are systematically ambiguous between a purpose and a reason interpretation, similarly to Mandarin, Russian, and Polish (contra Stepanov \& Tsai 2008). We argue that the distinct semantic interpretations correspond to two distinct base-generated positions of why. While reason why is base-generated within CP (Rizzi 2001, Ko 2005), purpose why is adjoined to $v \mathrm{P}$ (Stepanov \& Tsai 2008). Furthermore, we show that English purpose why, similarly to previously reported data from Mandarin, is only compatible with dynamic predicates with agentive subjects. We argue that this selectional restriction follows from two properties: (i) why semantically requires a proposition as its argument, and (ii) only dynamic predicates with agentive subjects have a syntactic structure that accommodates two adjunction sites of the relevant semantic type, i.e., they contain two distinct propositional levels (Bale 2007) and therefore two attachment sites for why. In contrast, propositionally simple predicates only have one propositional level and hence only one possible attachment site, which corresponds to the reason interpretation of why. Evidence for this proposal comes from the observation that only the lower why - associated with the purpose reading - is sensitive to negative islands, which suggests that its attachment site is below negation $(v \mathrm{P})$, whereas the higher why is insensitive to island effects of this sort, which suggests that its base generated position is above negation (CP).
\end{abstract}

Keywords. why-questions; propositional complexity; negative islands; modal obviation; downward entailing environment

1. Introduction. Cross-linguistically, why-questions have been shown to be ambiguous between a purpose and a reason interpretation. For example, Tsai $(1994,2008)$ and Stepanov \& Tsai (2008) demonstrate that both readings are available in Mandarin, as shown in (1) with the reason interpretation in (1a) and the purpose interpretation in (1b).

*Thank you to Susana Béjar, members of the Syntax Lab at McMaster University, and the audience at the annual meeting of the LSA 2016, especially Masaya Yoshida, for their comments and suggestions on this project. This research was supported by SSHRC grant \#435-2012-1567. C. Chapman's contribution was also partially supported by a SSHRC CGS doctoral scholarship \#767-2014-1827. Any remaining errors are our own. Authors: Cassandra Chapman, McMaster University (chapmc3@mcmaster.ca) \& Ivona Kučerová, McMaster University (kucerov@mcmaster.ca). 
a. Akiu weishenme chai-diao qiangbi?

Akiu why tear-down wall

'Why did Akiu tear down the wall?'

b. Akiu wei(-le) shenme chai-diao qiangbi?

Akiu for(-Prf) what tear-down wall

'For what purpose did Akiu tear down the wall?'

(Stepanov \& Tsai 2008:599)

This pattern has also been attested in other languages, including Russian and Polish (see Tsai 2008 and Stepanov \& Tsai 2008 for Russian and Jędrzejowski 2014 for Polish). In these languages, there are distinct lexical entries for reason and purpose why. However, Stepanov \& Tsai (2008) argue that English does not have a lexical entry for the purpose interpretation of why and thus, that English only allows the reason interpretation in why-questions. Previous work on English why also supports this claim. In most previous work, with the exception of Starke (2001), only the reason interpretation of why is ever discussed. ${ }^{1}$

We argue against previous analyses and show that English monoclausal why-questions are in fact systematically ambiguous between two readings, i.e., purpose and reason, similarly to Mandarin, Russian, and Polish. However, unlike these other languages, English does not have two distinct morphological forms for the two readings of why and therefore, why always appears in the same surface form. The distinction between the two readings can be made explicit in question-answer pairs, as in (2). ${ }^{2}$

$1 \quad$ Ambiguity in biclausal why-questions has, however, been well documented. Rizzi (2001) (also see Shlonsky \& Soare 2011) argues that biclausal why-questions in English are ambiguous between a short and long construal reading, as shown in (i). If the short construal reading is adopted, then why modifies the embedded verb, i.e., resign; the question asked about the reason for her resigning. However, if the long construal reading is adopted, then why modifies the matrix verb, e.g., ask; the question asks about the reason for asking. In both cases, the existing literature only considers a reason interpretation of why.

(i) Why did you ask her to resign?

Short construal:What is the reason, $x$, such that for $x$, you asked her to resign? e.g., Because I didn't want to just tell her.

Long construal: What is the reason, $x$, such that you asked her to resign for that particular reason, $x$ ? e.g., I asked her to resign because of her health, not because of her intelligence.

(Shlonsky \& Soare 2011:655)

2 We are not the first to discuss the interpretation differences in English why-questions. In his thesis, Starke (2001) argues that English why has two possible readings, which he calls "motivation" (or intentional cause) and "cause." The motivation reading corresponds to our purpose reading and the cause reading corresponds to our reason reading, as shown in (i):

(i) Context: Sissy woke up early in order to see the sunrise because she needed some comforting. Why did she wake up so early?

a. Purpose (motivation): In order to see the sunrise.

b. Reason (cause): Because she needed some comforting.

(Starke 2001:30) 
Why did she resign?

a. Purpose reading: For what purpose did she resign? (future event) In order to earn more money next year.

b. Reason reading: What was the reason for her resigning? (past event) Because she got a pay cut.

The purpose answer, e.g., (2a), always corresponds to a future-oriented event whereas the reason answer always corresponds to a past event, e.g., (2b). ${ }^{3,4}$

Interestingly, not all English predicates are compatible with both the reason and purpose interpretations. While why-questions with agentive dynamic predicates are systematically ambiguous between a purpose and a reason reading (see Tsai 1994, 2008, and Stepanov \& Tsai 2008 for a similar pattern in Mandarin), (3), only the reason reading is possible with non-agentive predicates (passives, unaccusatives, and locative-existential constructions), as in (4), ${ }^{5}$ (5), and (6). In other words, the purpose reading is incompatible with non-agentive predicates. ${ }^{6}$

\section{Dynamic predicate: both readings are possible}

Why did John tear down the wall?
a. $\checkmark$ Purpose: to build a newer one
b. $\quad \checkmark$ Reason: because he felt like it

Passive: only reason

Why was that student cheated out of a grade?

a. \#Purpose: to help him get a better grade on the final

b. $\checkmark$ Reason: because the instructor felt like it

3 We suggest that the interpretive distinction arises at the syntax-semantics interface. For reasons of space we cannot go into the details of the corresponding semantic analysis but the basic idea is that the reason attachment site is above the tense node: the presence of tense marking allows for a causal interpretation. In contrast, the attachment site for the purpose interpretation is below the tense node and, in turn, is not compatible with tense interpretations. However, the purpose reading may obtain a modal, that is, a future-oriented interpretation.

4 Our examples are constructed so that the purpose answer always appears in a non-finite clause beginning with in order to and the reason answer always takes the form of a because-clause. Although it is possible to have a finite purpose answer, speakers tend to accommodate a reason interpretation in these structures (see Stepanov \& Tsai 2008). The infinitival answer blocks the reason interpretation and, in turn, provides a clearer testing environment.

5 Kim et al. (2015) argue that when a predicate has an implicit agent, as is the case in a passive construction, the reason reading is infelicitous (their judgment):

(i) Why was the ship sunk?

a. In order to collect the insurance money.

b. \#Because they wanted to collect the insurance money.

We do not agree with this judgement but we leave it for future work to determine if the difference in judgements might depend on speaker variation.

6 A similar conclusion was reached by Kim et al. (2015). Thanks to Masaya Yoshida for bringing this work to our attention. 
a. ??to make more room in our fridge

b. $\checkmark$ because I left it out in the sun

Locative-existential predicate: only reason

Why are there three engineers on the NSF linguistics committee?

a. ??to block funding of theoretical research

b. $\checkmark$ because they were asked to help out

This leads us to ask: Why are only dynamic predicates compatible with both readings? In order to address this question, we need to first determine how the two distinct readings arise syntactically. We follow previous literature and argue that the two different readings of why correspond to two distinct base-generated positions in the syntax, namely spec-CP (reason) and spec- $v \mathrm{P}$ (purpose). Thus, our original question can be rephrased as: why do only certain predicates allow both attachment sites? We argue that the restriction directly stems from the semantic properties of the predicates. We follow Bale (2007) in arguing that only dynamic predicates are propositionally complex. More precisely, only dynamic predicates contain two syntactic levels that semantically correspond to a complete proposition. ${ }^{7}$ Since why semantically modifies a proposition, ${ }^{8}$ its sister must be of a propositional type. In turn, since only dynamic predicates have two syntactic levels of the appropriate semantic type, only dynamic predicates allow two distinct attachment sites for why. In contrast, non-agentive predicates are propositionally simple, and thus only allow one attachment site for why.

If the distinction between the two readings is indeed structural, that is, if there are two hierarchically distinct attachment sites corresponding to the two distinct readings of why, we should be able to detect the hierarchical difference. As we will see, our analysis correctly predicts that purpose why is sensitive to negation as it is base-generated below Neg, while reason why shows no negative island effect as it is base-generated above Neg. Moreover, our data provide additional evidence for a semantic analysis of weak islands (contra Relativized Minimality, Rizzi 1990): as we will demonstrate, the weak island can be obviated by an existential modal and the purpose reading is also sensitive to downward entailing environments.

2. Proposal. We argue that the two distinct interpretations correspond to two different syntactic structures. More precisely, we argue that why can attach to two distinct positions in the syntax. While reason why is based-generated in CP (Hornstein 1995, Rizzi 1990, 2001, Starke 2001, Ko 2005, Stepanov \& Tsai 2008, Thornton 2008), ${ }^{9}$ purpose why is generated as adjoined to $v \mathrm{P}$ (Starke 2001, Tsai 2008, Stepanov \& Tsai 2008). ${ }^{10}$ This predicts that in order to get the purpose interpretation, there needs to be a position to which why can attach in the $v \mathrm{P}$ domain. As

$7 \quad$ We use the term "proposition" in a technical sense. That is, for us, a proposition can be tenseless.

8 Rather informally, the denotation of a why-question is the set of alternative propositions that give the reason or purpose as to why $p$ is true.

9 Kim et al. (2015) argue that reason why originates in spec-TP and moves to spec-CP. For simplicity, we follow previous literature and assume that why is base-generated in its surface position, i.e., spec-CP, but as far as we can tell, nothing in the proposal hinges on this simplification.

10 Collins (1991) observes that why is permitted in tenseless clauses, unlike other wh-adjuncts: 
mentioned in the Introduction, only certain predicates, namely agentive predicates, are compatible with both interpretations. Thus, according to our analysis, only agentive predicates have both attachment sites available for why. This leads us to ask: why are both attachment sites not available with non-agentive predicates? In other words, why is the purpose reading infelicitous with non-agentive predicates?

2.1 BALE (2007) To explain these facts, we need to first look at the domain in which why attaches in the structure. As why always modifies a proposition, we argue that it needs to attach to a propositional level, i.e., it needs a proposition as its sister. This predicts that if there is more than one possible interpretation of why and therefore more than one attachment site for why, then there must be more than one propositional level in the structure. We follow Bale (2007) in arguing that predicates come in different flavors and can differ in their propositional complexity. More precisely, some predicates are not propositionally complex and have only one propositional level while others are propositionally complex and have two propositional levels. Under Bale (2007)'s analysis, only non-stative, transitive predicates, e.g., hit, are propositionally complex and have two propositional levels whereas intransitive, e.g., dance, and stative transitive verbs, e.g., hate, are propositionally simple and contain only one propositional level. Bale (2007) argues that non-stative transitive verbs are propositionally complex because they take the direct object as their argument but the subject is external to the verb. Thus, the verb and its object form one propositional level and the VP and its subject form a second propositional level. Importantly, the second propositional level does not include the subject. In contrast, as there is only one propositional level in intransitive and stative transitive verbs, that propositional level must include the subject.

To test for propositional complexity, Bale (2007) uses the adverb again, which he argues must syntactically adjoin to a propositional level and carries a presupposition that includes only its complement. This predicts that the presupposition associated with again must include the subject for propositionally simple predicates (intransitive and stative transitives) but that it does not need to include the subject for propositionally complex predicates (non-stative transitives). Bale argues that when again appears at the beginning of the sentence, then the presupposition associated with again must necessarily involve the subject. However, if again appears to the right of the VP, then the presupposition does not need to involve the subject. The prediction is that presuppositions not involving the subject should only be possible with propositionally complex predicates and that they should be impossible with propositionally simple predicates. Recall that when the predicate is propositionally complex, then it has two propositional levels, only one of which includes the subject. This prediction is borne out for non-stative transitive verbs, as shown in (7).

(i) a. Why go to the store, when there is orange juice at home?

b. *How come/when go to the store (when there is orange juice at home)?

(Adapted from Collins 1991:34)

Collins (1991) does not provide an analysis to explain why this is the case. Yoshida et al. (2015) suggest that tenseless clauses are a type of why-stripping in which the VP is a non-wh-remnant. 
(7) Jon and his wife love their daughter, Esme, and Esme is reassured by overt expressions of their love. For example, yesterday Esme felt reassured when her mother gave her a hug. The effect was doubled when...

a. Jon hugged her again.

b. \#Again Jon hugged her.

c. She was hugged again.

(Bale 2007:463)

In (7a), the presupposition associated with again is satisfied by the prior context: Jon does not need to have hugged Esme before; she could have been hugged by someone else, provided that she was hugged before. This presupposition is also satisfied in (7c). The presupposition in (7b) must include Jon and this presupposition is not satisfied by the prior context and is thus infelicitous. Thus, presuppositions not involving the subject are felicitous with non-stative transitive verbs, providing evidence that these predicates have an additional propositional level that does not include the subject. In contrast, the presupposition associated with again in stative transitive and intransitive verbs (propositionally simple), cannot exclude the subject, as shown in (8) for stative transitive, hate.

(8) Seymour's sister hated George. But she seemed to be the only one who did. After a while George worked his charm on her and the hatred subsided. After a few months, Seymour realized that George's charm was all an act. Underneath, he was pure evil. So...

a. \#Seymour hated George again.

b. \#Again Seymour hated George.

c. George was hated again.

(Bale 2007:469)

In both (8a) and (8b), the presupposition is not compatible with the previous context because Seymour did not hate George before. Therefore, the presupposition cannot exclude the subject, whether again follows (8a) or precedes (8b) the VP. However, the presupposition in (8c) is compatible with the context because George was hated before. Thus, only the presupposition in the passive structure is compatible with the context. These examples demonstrate that in contrast to the non-stative transitive in (7), the presupposition in stative transitives must necessarily include the subject, ${ }^{11}$ providing evidence that there is only one propositional level in stative transitive verbs.

2.2 EXTENDING BALE (2007) TO THE CURRENT STUDY As described in the previous sub-section, Bale (2007) uses the presupposition associated with again to test whether a predicate is propositionally complex. If a predicate has two propositional levels, then it should allow a presupposition that does not include the subject but if a predicate has only one propositional level, then the presupposition should necessarily include the subject. The proposal that dynamic, agentive predicates are propositionally complex is borne out, as shown in (9).

$11 \quad$ Similar effects are found with intransitive verbs. See Bale (2007) for further details. 
John received mixed information from the city about whether he needed to have a wall in place to put a pool in his backyard. He first heard that he didn't need the wall so he called a contractor to come and tear it down. Then he found out that he needed to have the wall so he asked the contractor to come and put it back up. Most recently, he found out that he does not in fact need the wall. To save money:

a. John tore down the wall again.

b. \#Again John tore down the wall.

c. The wall was torn down again.

The context does not support the presupposition in (9b): John was not the one who tore down the wall before. In contrast, the presupposition holds in (9a) and (9c) because the wall was indeed torn down before. This test provides evidence that the dynamic predicates used for the current study are propositionally complex and have two propositional levels. Unfortunately, the presupposition test cannot be used with the non-agentive predicates. The crucial prediction is that the presupposition must include the subject for propositionally simple predicates. As our non-agentive predicates already do not have external subjects, we cannot use this test.

2.3 More THAN ONE ATTACHMENT SITE We propose that only propositionally complex predicates can have two attachment sites for why: spec-CP and spec- $v \mathrm{P}$. As they are propositionally complex, they have two propositional levels and thus allow both readings of why. In contrast, non-agentive predicates only have one propositional level and in turn, they only have one attachment site for why, i.e., spec-CP. This correctly predicts that non-agentive predicates are only compatible with the reason interpretation but that dynamic predicates allow both readings. ${ }^{12}$

In other words, in order to get the purpose reading, there needs to be a more complex structure that includes a $v \mathrm{P}$ level. For concreteness, we link the additional propositional level to an additional functional level in $v$ P, namely CauseP (Kratzer 1996, Marantz 1997). CauseP has been argued to correspond to agentive predicates having a volitional requirement. According to Kratzer (1996), the Agent is introduced by a voice projection, or CauseP. Crucially, predicates with which the purpose reading is not felicitous have no agent, i.e., unaccusatives, passives, and locative existentials. We propose that purpose why attaches to the $v \mathrm{P}$ level but that it can only do so if there is an expanded VP. More precisely, we assume that the Cause projection is introduced by the agentive root, which further explains why we lose the purpose reading with non-agentive predicates (Marantz 1997). We propose the structure in (10), in which Reason why adjoins to the higher $\mathrm{CP}$ and purpose why adjoins to $v \mathrm{P}$. Note that CauseP constitutes one propositional level while CP constitutes the second propositional level.

12 The propositional requirement also accounts for the non-existence of whyever adverbials in English and other distributional facts observed in Stepanov \& Tsai (2008). -ever is a free choice function morpheme that requires an existential closure of some sort: in turn, it is semantically incompatible with why that selects for a proposition. 


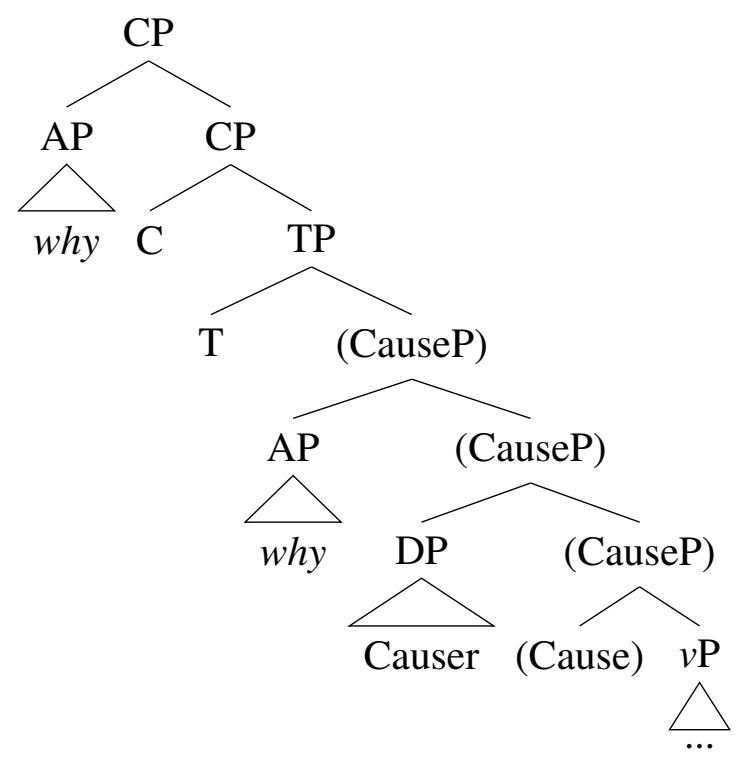

3. Predictions. The proposal that the two types of why adjoin to different levels in the structure makes three empirical predictions, which we will show are all borne out. We have argued that purpose why is unavailable with non-agentive predicates because these predicates are propositionally simple. Thus, our first prediction is that creating a more complex structure should make the purpose reading available. The second prediction is that negation should be an intervener for the purpose reading but not for the reason reading. As purpose why adjoins to $v \mathrm{P}$, it adjoins below negation. In contrast, as reason why adjoins to CP, it adjoins above negation. Therefore, purpose why but not reason why should be sensitive to negative intervention effects. We will show that this prediction is borne out but we argue that the negative island effect is a weak island effect and not attributable to Relativized Minimality. The evidence for this comes from our third prediction: the weak island should be obviated by existential modals but the weak island effect should reappear with other downward entailing elements, e.g., few.

3.1 Prediction 1: More COMPlex StRuCture Allows Both REAdings On the surface, the purpose reading seems to be a less complex structure because it always consists of an infinitive structure. This suggests that a simplex $v \mathrm{P}$ structure does not yield the purpose reading. Wurmbrand $(2001,2007)$ argues that infinitives can be of different syntactic sizes. More precisely, the structural size of an infinitive depends on the structural material that has been merged. This predicts that if we add more structural material to the $v \mathrm{P}$, then we will create a more complex $v \mathrm{P}$, which should yield a purpose reading. As shown in (11), (12) and (13), by increasing the size of the $v \mathrm{P}$ using for-infinitives, the purpose reading becomes available.

Passive

Why was that student hit?

a. \#to help him get an easier opponent (tomorrow)

b. $\quad \checkmark$ for him to get an easier opponent (tomorrow) 
a. \#to make the party more lively

b. $\quad \checkmark$ for the party to be more lively

\section{Locative-existential}

Why are there three engineers on the NSF linguistics committee?

a. \#to encourage funding for theoretical linguistics

b. $\quad \checkmark$ for more funding to be distributed to theoretical linguists

3.2 Prediction 2: Negation Unlike other adjunct wh-phrases, English why-questions do not produce a negative island effect in mono-clausal environments, as shown in (14) (see e.g., Beck 2006). Nevertheless, they are sensitive to negation if why undergoes long-distance $w h$-movement (Rizzi 1990, 2001, Shlonsky \& Soare 2011; cf. ft. (i)), (15). When embedded under negation, the long construal reading is unavailable (Rizzi 1990, 2001), i.e., it can only question your reason for not asking her to resign. This asymmetry has been explained by the structural position of why, i.e., whether why is base-generated above or below negation.

a. Why didn't Peter come?

b. *How didn't Peter drive?

a. Why did you say that Mary resigned?

$\checkmark$ the reason for saying

$\checkmark$ the reason for resignation

b. Why didn't you say that Mary resigned?

$\checkmark$ the reason for not saying

\# the reason for resignation

The fact relevant to us is that we also see an effect of negation in monoclausal why-questions. While the sentence may seem grammatical on the surface, we expect only one of the interpretations to be available in negative why-questions, namely, the reason reading. This prediction is borne out based on the structural analysis we have proposed. Since the two readings of why correspond to two different structures, they should show different sensitivity to negation. Reason why is adjoined to $\mathrm{CP}$ and is thus situated above negation, predicting that it should not be sensitive to a negative intervener. In contrast, purpose why is adjoined to $v \mathrm{P}$ and is thus generated below negation. It should therefore be sensitive to a negative intervener. This is borne out, as shown by the contrast between (16) and (17), and (18) and (19).

(16) Why did John tear down the wall?

a. Reason: $\checkmark$ because he wanted to show off

b. Purpose: $\checkmark$ to build a newer one 
(17) Why didn't John tear down the wall?

a. Reason: $\checkmark$ because he didn't want to show off

b. Purpose: \#to put in a pool

(18) Why did you kiss Mary?

a. Reason: $\checkmark$ because I like her.

b. Purpose: $\checkmark$ to protect her.

(19) Why didn't you kiss Mary?

a. Reason: $\checkmark$ because I don't like her.

b. Purpose: \#to protect her.

Starke (2001) also provides evidence from negation to argue that the difference between the two readings of why-questions can be explained by a structural account, as demonstrated in (20).

(20) Brutus didn't go to Rome because his wheel broke.

a. Brutus didn't go to Rome and the reason for this is that his wheel broke.

b. Brutus did go to Rome and the reason for this is unrelated to the fact that his wheel broke.

(Starke 2001:30-31)

The construction in (20b) must be interpreted as a motivation (purpose) reading, e.g., Brutus went to Rome in order to visit the coliseum. In contrast, (20a) is ambiguous; it can either be interpreted as motivation (purpose) or cause (reason). Thus, the low scope reading (below negation) corresponds to the purpose reading (motivation) and the high scope reading (above negation) corresponds to the reason reading (cause). We argue that the negative island effect arises because purpose why would need to cross Neg when it raises to spec-CP and this movement is illicit, as schematized in (21). 


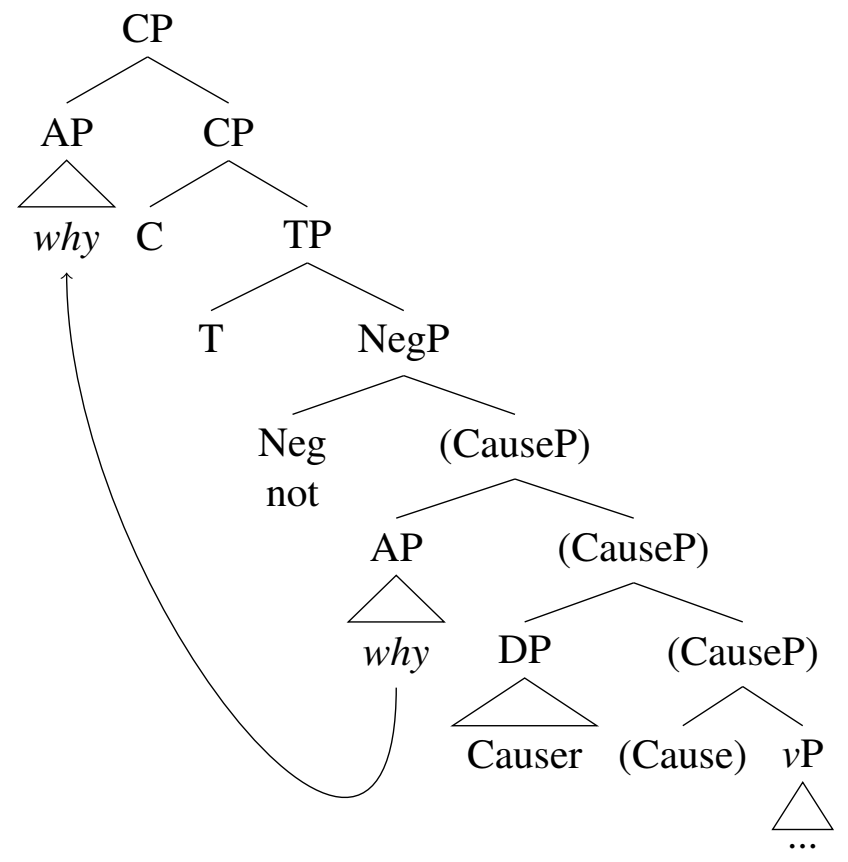

We argue that the negative intervention effect in purpose why-questions can be explained as a weak island, and not based on Relativized Minimality (contra Rizzi 1990, 2001). In other words, it results from the semantics of why-questions (see e.g., Dotlačil \& Nouwen 2016, Beck \& Rullmann 1999). We present specific evidence for this proposal in the next section. Before doing so, note that further evidence for the locality effect is provided in Starke (2001), as shown in (22).

a. Why did Sissy wake up early in order to see the sunrise? Because she needed some comforting!

b. *Why did Sissy wake up early because she needed some comforting? In order to see the sunrise!

(Starke 2001:31)

While the reason interpretation is compatible with an in order to statement in the same clause, (22a), purpose why is not compatible with a because statement occurring in the clause, (22b). These data provide further evidence that purpose why starts lower in the structure and moves to the left periphery. More precisely, as mentioned by Starke (2001), adjunct wh-movement generally cannot move over because-clauses, as shown in (23), providing further evidence in favor of the locality account. 
a. *How did Sissy behave because she likes him?

b. *When did Sissy wake up because she was sad? At 6:00 in the morning.

c. ?What did Sissy buy because she was feeling blue ${ }^{13}$

(Starke 2001:31)

\subsection{PREDiCTION 3: MOdAl OBVIATION AND DOWNWARd ENTAILING ENVIRONMENTS We} follow Beck \& Rullmann (1999) in arguing that negative island effects in general arise due to a difficulty with resolving the interpretation of the wh-element and, in turn, we expect the $w h$-element to be sensitive to other structural elements that affect the interpretation of wh-questions. Previous work on weak islands has shown that existential modals can obviate weak islands but that universal modals cannot (Abrusán 2007, Abrusán \& Spector 2011, Fox \& Hackl 2006). If the negative island effect presented in the last section is a type of weak island effect, then it should also be possible to obviate the island with an existential modal. As we see in (24), this prediction is borne out: in negative environments, the purpose reading is possible with an existential modal but not with a universal modal.

(24) Context: To put in a pool in one's backyard, there must be a wall in place ${ }^{14}$. Why didn't John tear down the wall?

a. Existential: $\checkmark$ to be allowed to have a pool

b. Universal: \# to be required to have a pool

Further evidence comes from the behavior of the purpose reading in other downward entailing environments. If the negative island is a type of weak island, then we expect the purpose reading to also disappear in other downward entailing environments, e.g., under the scope of the quantifier few. This is borne out, as demonstrated by the contrast between (25) and (27) on the one hand and (26) and (28) on the other hand.

(25) Why did the professors attend the party?

a. Purpose: $\checkmark$ to get to know the graduate students

b. Reason: $\checkmark$ because they thought it would be fun

(26) Why did few professors come to the Christmas party?

a. Purpose: \#to eat all the snacks

b. Reason: $\checkmark$ because they thought it would be boring

(27) Why did the boys kiss Mary?

a. Purpose: $\checkmark$ to annoy her

b. Reason: $\checkmark$ because they like her

13 Starke (2001) also provides evidence that reason why does not move in the structure but we leave these data out due to space restrictions. See Starke (2001) for more details.

14 Thanks to Susana Béjar for her help with constructing this example. 
a. Purpose: \#to protect her

b. Reason: $\checkmark$ because they don't like her

Similarly to the negative island examples, the purpose reading is infelicitous when why is embedded under the scope of $f e w$. These data would not be predicted by a Relativized Minimality account but straightforwardly follow from a weak island account.

5. Conclusions. We have argued that English monoclausal why-questions are systematically ambiguous between a reason and a purpose interpretation, similarly to Mandarin, Russian, and Polish. Unlike these other languages, English does not have distinct morphological forms for each reading of why but instead, the difference in interpretation can clearly be detected in question-answer pairs of the why-question. We argued that reason why adjoins to CP whereas purpose why adjoins to $v \mathrm{P}$. We demonstrated that only propositionally complex predicates are compatible with both readings of why. Propositionally simple sentences do not permit the purpose interpretation of why. Following Bale (2007), we argued that propositionally complex predicates have two propositional levels whereas propositionally simple predicates only have one propositional level. The additional propositional level in dynamic predicates creates a second adjunction site for why, allowing the purpose reading. This proposal predicted that increasing the complexity of the $\nu \mathrm{P}$ in non-agentive predicates should make the purpose reading available. Using for-infinitives, we demonstrated that this prediction is borne out. Further evidence for our proposal was provided by data showing that only the purpose reading is sensitive to a negative intervener. We argued that this effect is a type of weak island in English. Support for this proposal was provided by modal obviation data, demonstrating that the purpose reading survives under negation with existential modals, but not with universal modal, and from downward entailing environments, demonstrating that the purpose reading also disappears under the scope of few. We therefore argued that the negative island effect can be explained by a semantic analysis of weak islands, and not by Relativized Minimality. To conclude, this paper has shown that English why-questions show a similar interpretation ambiguity to languages with richer morphology. In order to access both readings of why, the answer to the question needed to be carefully controlled.

\section{References}

Abrusán, Márta. 2007. Contradiction and grammar: The case of weak islands. Cambridge, MA: MIT dissertation.

Abrusán, Márta \& Benjamin Spector. 2011. A semantics for degree questions based on intervals: Negative islands and their obviation. Journal of Semantics 28(1). 107-147. http://dx.doi.org/10.1093/jos/ffq013

Bale, Alan Clinton. 2007. Quantifiers and verb phrases: An exploration of propositional complexity. Natural Language \& Linguistic Theory 25(3). 447-483. http://dx.doi.org/10.1007/s11049-007-9019-8

Beck, Sigrid. 2006. Intervention effects follow from focus interpretation. Natural Language Semantics 14(1). 1-56. http://dx.doi.org/10.1007/s11050-005-4532-y 
Beck, Sigrid \& Hotze Rullmann. 1999. A flexible approach to exhaustivity in questions. Natural Language Semantics 7(3). 249-298. http://dx. doi .org/10.1023/A:1008373224343

Collins, Chris. 1991. Why and how come. In Lisa Lai Shen Cheng \& Hamida Demirdash (eds.), More papers on wh-movement: MIT Working Papers in Linguistics 15. 31-45. Cambridge, MA: MIT Press.

Dotlačil, Jakub \& Rick Nouwen. 2016. The comparative and degree pluralities. Natural Language Semantic 24(1). 45-78. http: //dx . doi .org/10.1007/s11050-015-9119-7

Fox, Danny \& Martin Hackl. 2006. The universal density of measurement. Linguistics and Philosophy 29(5). 537-586. http://dx .doi .org/10.1007/s10988-006-9004-4

Hornstein, Norbert. 1995. Logical form: From GB to minimalism. Cambridge: Blackwell.

Jędrzejowski, Łukasz. 2014. Again on why. but why? In Cassandra Chapman, Olena Kit \& Ivona Kučerová (eds.), Formal Approaches to Slavic Linguistics 22: The McMaster Meeting 2013. 151-169. Ann Arbor: Michigan Slavic Publications.

Kim, Nayoun, Masaya Yoshida \& Alexis Wellwood. 2015. Two whys in English. Talk presented at SWAMP 2015. University of Michigan.

Ko, Heejeong. 2005. Syntax of why-in-situ: Merge into [Spec, CP] in the overt syntax. Natural Language \& Linguistic Theory 23(4). 867-916. http://dx.doi.org/10.1007/s11049-004-5923-3

Kratzer, Angelika. 1996. Severing the external argument from its verb. In Johan Rooryck \& Laurie Zaring (eds.), Phrase structure and the lexicon. 109-137. Dordrecht: Kluwer Academic Publishers.

Marantz, Alec. 1997. No escape from syntax: Don't try morphological analysis in the privacy of your own lexicon. In Alexis Dimitriadis, Laura Siegel, Clarissa Surek-Clark \& Alexander Williams (eds.), Proceedings of the 21st Annual Penn Linguistics Colloquium, University of Pennsylvania Working Papers in Linguistics 4.2. 201-225.

Rizzi, Luigi. 1990. Relativized Minimality.. Cambridge, MA: MIT Press.

Rizzi, Luigi. 2001. On the position "Int(errogative)" in the left periphery of the clause. In Guglielmo Cinque \& Giampaolo Salvi, Current studies in Italian syntax: Essays offered to Lorenzo Renzi. 267-296. Amsterdam: Elsevier.

Shlonsky, Ur \& Gabriela Soare. 2011. Remarks and replies: Where's 'why'? Linguistic Inquiry 42(4).651-669. http://dx.doi.org/10.1162/LING_a_00064

Starke, Michal. 2001. Move dissolves into merge: A theory of locality. Geneva: University of Geneva dissertation. http://ling. auf . net/lingbuzz/000002

Stepanov, Arthur \& Wei-Tien Dylan Tsai. 2008. Cartography and licensing of wh-adjuncts: A cross-linguistic perspective. Natural Language \& Linguistic Theory 26(3). 589-638. http://dx.doi.org/10.1007/s11049-008-9047-z

Thornton, Rosalind. 2008. Why continuity. Natural Language \& Linguistic Theory 26(1). 107-146. http://dx.doi.org/10.1007/s11049-007-9031-z

Tsai, Wei-Tien Dylan. 1994. On nominal islands and LF extraction in Chinese. Natural Language \& Linguistic Theory 12(1). 121-175.

Tsai, Wei-Tien Dylan. 2008. Left periphery and how-why alternations. Journal of East Asian Linguistics 17. 83-115. http://dx.doi.org/10.1007/s10831-008-9021-0

Wurmbrand, Susanne. 2001. Infinitives: Restructuring and clause structure. Berlin: Mouton de Gruyter. 
Wurmbrand, Susi. 2007. Infinitives are tenseless. In Tatjana Scheffler, Joshua Tauberer, Aviad Eilam \& Laia Mayol (eds.), Proceedings of the 30th Annual Penn Linguistics Colloquium, University of Pennsylvania Working Papers in Linguistics 13.1. 407-420.

Yoshida, Masaya, Chizuru Nakao \& Ivan Ortega-Santos. 2015. The syntax of why-stripping. Natural Language \& Linguistic Theory 33(1). 323-370.

http://dx.doi.org/10.1007/s11049-014-9253-9 